\title{
Wire-guided stricturotomy for sealed ileal pouch
}

A 31-year-old woman with a 10-year history of ulcerative colitis was seen at our outpatient clinic with complaint of abdominal pain and increased output from her ileostomy. She had previously undergone a subtotal colectomy with an end ileostomy for medically refractory ulcerative colitis in 2008. She then underwent elective complete proctectomy with ileal pouch-anal anastomosis and diverting loop ileostomy 6 months later. We decided to perform pouchoscopy to rule out pouch-related complications ( $>$ Video 1 ).

Illeoscopy was performed via the stoma. The terminal ileum appeared normal to $25 \mathrm{~cm}$ from stoma. The patient was found to have a diverted pouch outlet stricture. The sealed outlet was detected by a Jag-

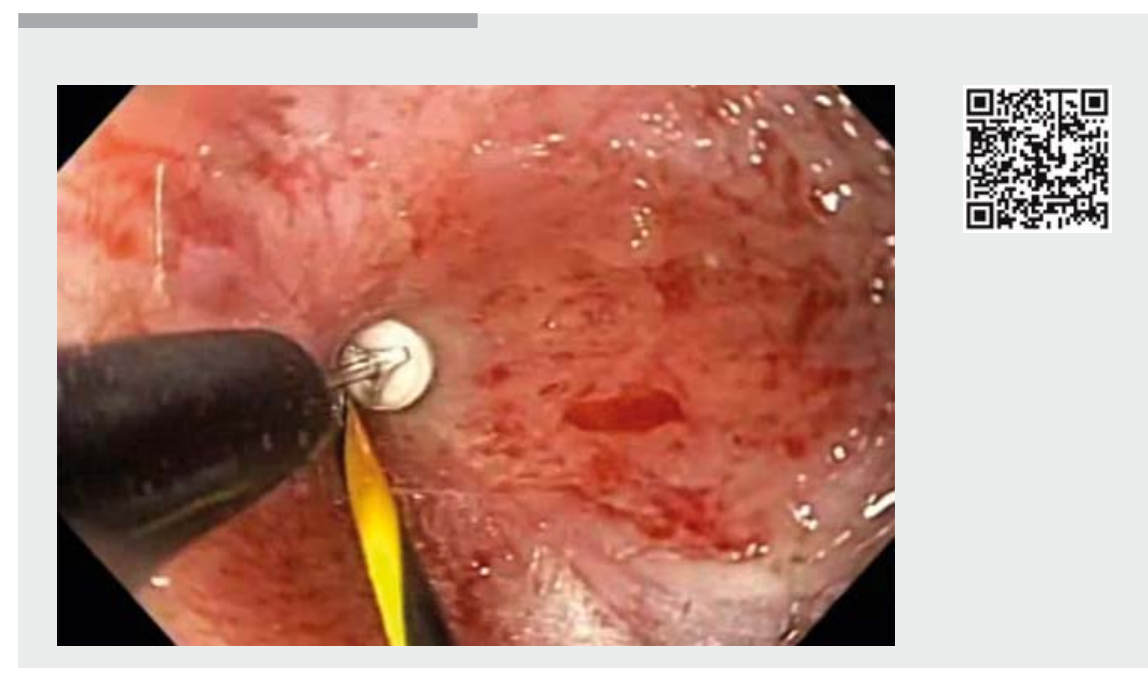

$\checkmark$ Video 1 Wire-guided stricturotomy for sealed ileal pouch.
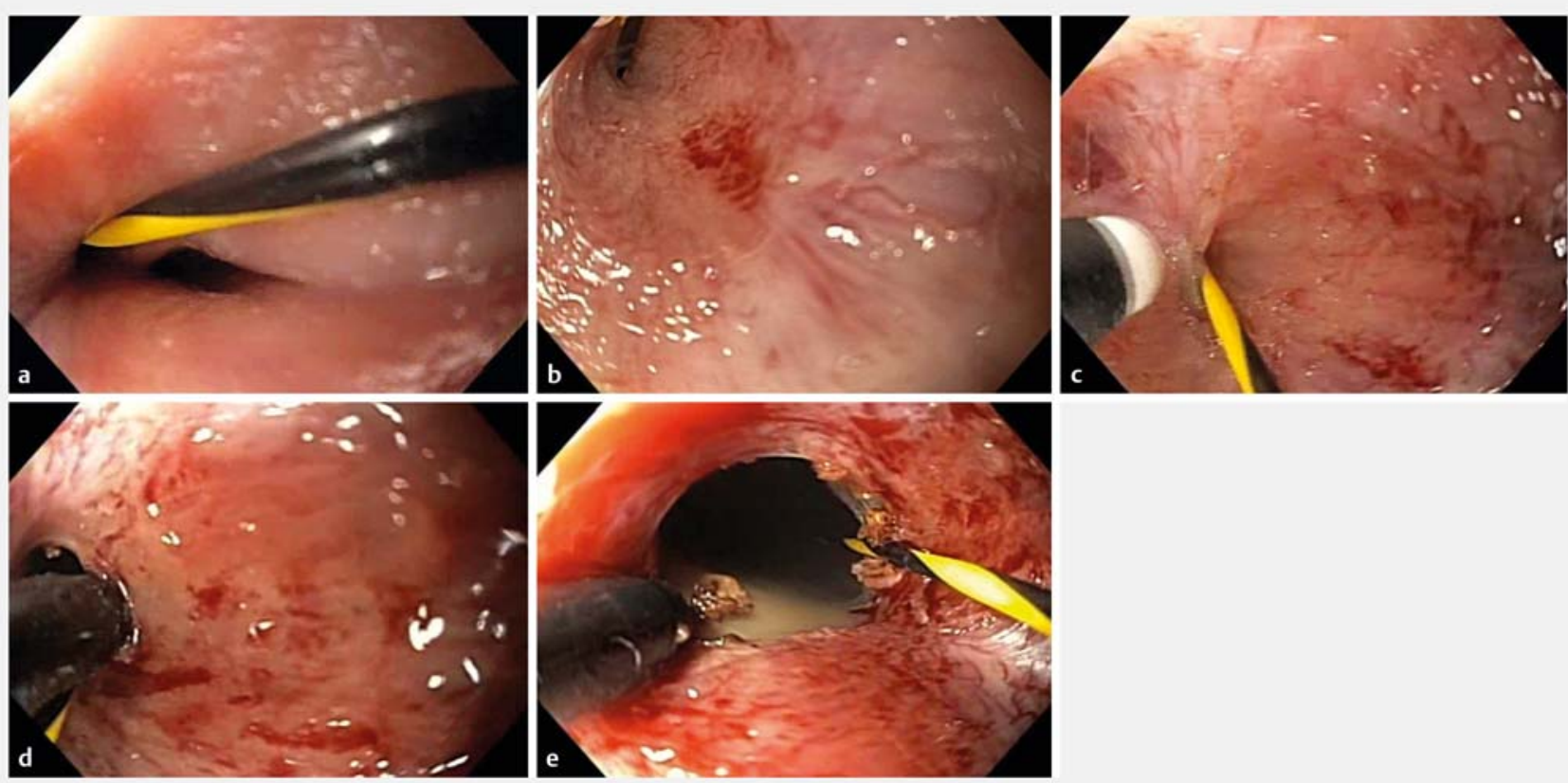

- Fig. 1 Wire-guided stricturotomy of sealed ileal pouch. a Sealed pouch outlet was detected by a Jagwire (Boston Scientific, Marlborough, Massachusetts, USA). b Diverted pouch outlet stricture was noted. c Knife stricturotomy was performed over the guidewire. $\mathbf{d}$ Knife stricturotomy was performed in a radial fashion. e Sealed ileal pouch was effectively treated with wire-guided stricturotomy. 
wire (Boston Scientific, Marlborough, Massachusetts, USA) (ฉ Fig.1a,b). We then performed knife stricturotomy over

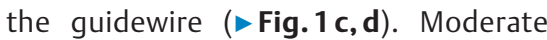
diversion pouchitis with exudates was noted. We were able to pass the scope without difficulty ( Fig. 1 e).

The patient tolerated the procedure well without any immediate complications. She reported improvement in her symptoms at the 1-month follow-up visit. Sealed ileal pouch can be safely and effectively treated with wire-guided endoscopic stricturotomy.

Endoscopy_UCTN_Code_TTT_1AQ_2AF

Competing interests
The authors

\section{Freeha Khan, Bo Shen}

Center for Inflammatory Bowel Disease,

Digestive Disease and Surgical Institute,

Cleveland Clinic, Cleveland, Ohio, United States

Corresponding author

Bo Shen, MD

Center for Inflammatory Bowel Disease, Digestive Disease and Surgery Institute-A31, Cleveland Clinic, 9500 Euclid Ave., Cleveland, $\mathrm{OH} 44195$, United States Fax: +1-216-444-6305

shenb@ccf.org

\section{Bibliography}

DOI https://doi.org/10.1055/a-0840-3262

Published online: 12.2.2019

Endoscopy 2019; 51: 493-494

(c) Georg Thieme Verlag KG

Stuttgart · New York

ISSN 0013-726X

\section{ENDOSCOPY E-VIDEOS \\ https://eref.thieme.de/e-videos}

回陨 Endoscopy E-Videos is a free access online section, reporting 回嗮: on interesting cases and new techniques in gastroenterological endoscopy. All papers include a high quality video and all contributions are freely accessible online.

This section has its own submission website at https://mc.manuscriptcentral.com/e-videos 